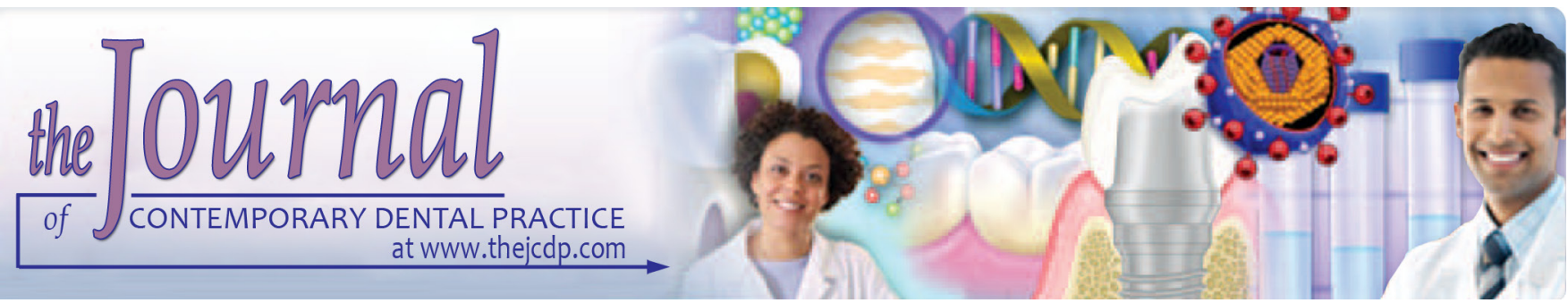

\title{
Evaluation of Radiation-induced Cytological Changes in Lesional Oral Cancer Cells and Adjacent Normal Mucosal Cells
}

\author{
${ }^{1}$ Vinit Patil, ${ }^{2}$ Rajendra Baad, ${ }^{3}$ Anand Gudur, ${ }^{4}$ Nupura Vibhute, ${ }^{5} \mathrm{Uzma}$ Belgaumi, ${ }^{6}$ Vidya Kadashetti
}

\begin{abstract}
Aim: To assess various cytological changes for predicting radiosensitivity of oral squamous cell carcinoma by exfoliative cytology.

Materials and methods: Histologically proven 30 cases of oral squamous cell carcinoma who underwent fractionated radiotherapy in a dose of 45-60 Gy in 5 fractions/week were enrolled in the study. The exfoliative cytology smear was evaluated on lesional and adjacent oral mucosa before radiotherapy, during radiotherapy (8 and 11th fraction) and post radiotherapy $(4,6$ and 8 weeks). Various parameters like multinucleation, cellular enlargement, nuclear enlargement, cytoplasmic vacuolation, cytoplasmic granulation, leukocytic infiltration were evaluated.

Results: Statistical significant values were seen in the intergroup comparison of all the parameters when compared adjacent mucosa and normal mucosa for leukocytic infiltration in pretreatment smear.
\end{abstract}

Conclusion: The study showed that radiation-induced cytological changes in oral squamous cell carcinoma have a significant dose-related increase. This dose-response relationship and the high intratumoral variations suggest that serial assay of these changes has potential use for radiosensitivity prediction.

Clinical significance: Radiosensitivity prediction can be evaluated by means of cytological smears in one stop crisis centre (OSCC) individuals subjected to fractionated radiotherapy by evaluating the cytological parameters.

Keywords: Cytology, Oral cancer, Radiosensitivity.

How to cite this article: Patil V, Baad R, Gudur A, Vibhute N, Belgaumi $U$, Kadashetti $V$. Evaluation of Radiation-induced

1,2,4-6 Department of Oral Pathology and Microbiology, School of Dental Sciences, Krishna Institute of Medical Sciences Deemed University, Karad, Telangana, India

${ }^{3}$ Department of Oncology, Krishna Institute of Medical Sciences Deemed University, Karad, Telangana, India

Corresponding Author: Vinit Patil, Department of Oral Pathology and Microbiology, School of Dental Sciences, Krishna Institute of Medical Sciences Deemed University, Karad, Telangana, India, e-mail: vinitp42@gmail.com
Cytological Changes in Lesional Oral Cancer Cells and Adjacent Normal Mucosal Cells. J Contemp Dent Pract 2018;19(12):1475-1480.

Source of support: Nil

Conflict of interest: None

\section{INTRODUCTION}

Exfoliative cytology is the microscopic examination of shed cells from an epithelial surface. ${ }^{1}$ It has been known for more than 100 years that cells removed from tumors can provide a clue to diagnosis, ${ }^{2,3}$ but it was not until Papanicolaou, ${ }^{4}$ and Traut $^{5}$ and others ${ }^{6,7}$ who explored the diagnostic potential of the fact that, exfoliative cytology can be extensively applied to the detection of cancer. ${ }^{8}$

Oral cancer is a serious and growing problem in many parts of the globe. More than $90 \%$ of oral malignancies in the upper aerodigestive tract are squamous cell carcinomas. ${ }^{9}$

As a standard treatment for oral squamous cell carcinoma radiotherapy is frequently used which can either be alone or in combination with surgery. ${ }^{10}$ Evaluation of radiation-induced cellular changes with a view to predict radiosensitivity has to led to interest many investigators as such changes were first documented in biopsied material in $1935 .{ }^{11} \mathrm{~A}$ cytologic test (the radiation response test) for this in "non-malignant appearing cells" from vaginal smears was developed in $1947 .^{12}$ Both success ${ }^{13}$ and failure ${ }^{14}$ were later found by others in cancer of cervix.

Evaluation of cytologic changes due to irradiation on oral mucosa was first described in 1957 and on oral cancer in $1959 .{ }^{15}$ Later, however, reports indicated no paucity of correlation of the changes either with dose or tumor radiosensitivity. ${ }^{16,17}$

By 1960s pyknosis, karyorrhexis, karyolysis, enlargement, multinucleation, and crenation of nuclear membrane were the nuclear morphological changes that were evaluated by 
cytology and later became well established. ${ }^{18}$ Cytologically, malignant as well as benign cells show identical changes, except for cancer cells depict significant hyperchromasia with the increased relative nuclear area, coarse irregularly distributed chromatin and irregular nuclear outlines. ${ }^{19,20}$ Whereas, cytoplasmic vacuolation, multinucleation, bizarre cell formation, leukocytic infiltration, nuclear enlargement with clumping of chromatin and wrinkling of the nucleus are few of the other cellular changes which have been described to occur following post radiotherapy. ${ }^{21}$

The estimation of radiosensitivity of individual tumors will be essential for planning the optimum radiation schedule for each patient and in choosing the treatment. ${ }^{22}$ Evaluating the cytological changes for the sensitivity of oral squamous cell carcinoma in patients undergoing radiotherapy will help us observe the cytological changes before, during and after radiation. It will also help predict the firmness of relationship amongst dose and duration of radiation therapy of these changes.

This study was undertaken to determine if serial cytological evaluation done before during and postradiotherapy, in oral squamous cell carcinoma patients can predict radiosensitivity or not.

\section{MATERIALS AND METHODS}

A total of thirty patients with histologically proven cases of oral squamous cell carcinoma who had reported to Department of Radiotherapy and Oncology and underwent fractionated radiotherapy in a dose of 45 to 60 Gy in 5 fractions/week (total 30 fractions), were enrolled in the study.

\section{Inclusion Criteria}

- Patients undergoing fractionated radiotherapy in a dose of 45 to 60 Gy in 5 fractions/week. (Total 30 fractions).

- Patients undergoing the only radiotherapy for oral squamous cell carcinoma and not been treated with other modalities, like chemotherapy/surgery, along with radiotherapy during the course of study.

\section{Exclusion Criteria}

- Patients having different radiation schedules from the above mentioned.

- Patients who fail to turn up for follow-up on the intervals of the study.

The OSCC patients were evaluated in the following schedule:

- Pretreatment Smear

- During radiotherapy 8th fraction

- During radiotherapy 11th fraction

- Four weeks post-radiotherapy
- Six weeks post-radiotherapy

- Eight weeks post-radiotherapy

At each visit, the patients were asked to rinse their mouth scrupulously and following that the material was collected from the oral cavity by scarping the lesional site and the adjacent mucosa with a sterile wooden spatula which was moistened in distilled water to avoid discrepancies. Normal appearing mucosa just adjacent to the lesional site was considered in the study. For the preparation of smear during every visit two new sterile wooden spatulas were used, one for the lesional site and other for adjacent mucosa. The study included analysis of total six smears from OSCC lesional site and six smears from adjacent mucosa. The obtained materials were directly smeared on clean glass slides (different slides were used for lesional and adjacent mucosa). Further, immediately fixed in cytofixative solution, while they were wet, and sent to the laboratory for staining. These fixed slides were stained with Papanicolaou stain.

\section{Analysis of the Smears}

A total of 500 cells were evaluated by a single observer from the samples collected on each occasion. Smears were examined at both 20x and 40x with an eyepiece of 10x of a light microscope. The nuclear and cytoplasmic changes observed were: Nuclear enlargement, cellular enlargement, multinucleation, cytoplasmic vacuolation, cytoplasmic granulation, and leukocytic infiltration. Cell clumps, cells with indistinct nuclear membranes and poorly stained cells were not counted.

\section{RESULTS}

\section{Statistical Analysis}

Data was gathered, categorized and coded. Data were analyzed using SPSS-16 software using Independent t-test and repeated measures analysis of variance (ANOVA) test.

Graph 1 reveals the frequency distribution of gender among study. Out of 30 individuals enrolled there were 20 male and 10 female patients.

Table 1 reveals descriptive statistics for age in the study group with a mean age of 57.37 years.

In the present study, the cytological evaluation of the lesional and the adjacent mucosal site collected at various intervals was considered in the groups.

Thus, two major groups based on smear collection are:

- Lesional Mucosa

- Adjacent Mucosa

Tables 2 to 6 reveals intergroup comparison of multinucleation, nuclear enlargement, cellular enlargement, cytoplasmic vacuolation, cytoplasmic granulation scores 
among lesional mucosa and adjacent mucosa by Independent t-test. Amongst each group (adjacent mucosa group and lesional group) intragroup comparison for cellular

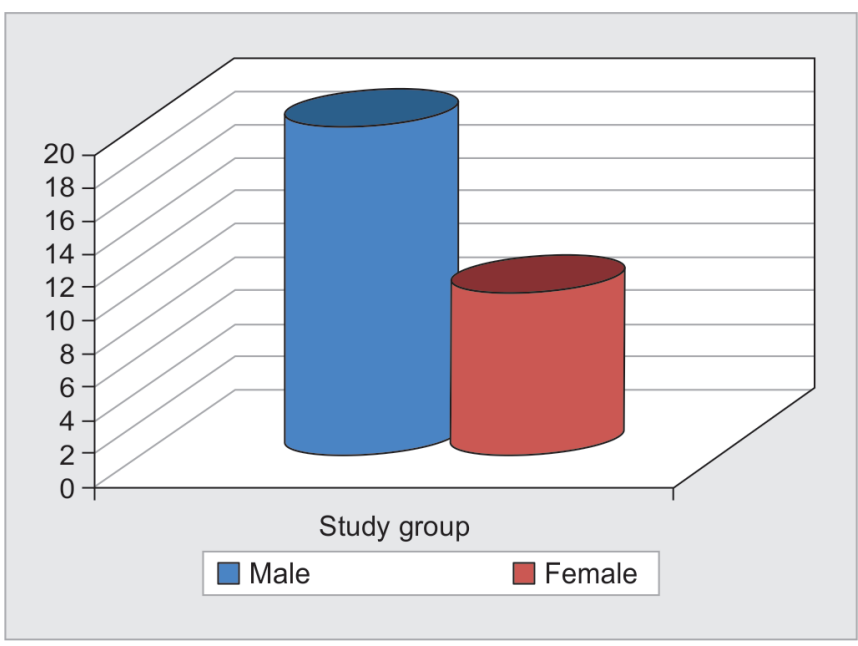

Graph 1: Frequency distribution of gender among study

Table 1: Descriptive statistics for Age in the study group

\begin{tabular}{llllll}
\hline \multicolumn{6}{c}{ Descriptive statistics } \\
\hline & $N$ & Minimum & Maximum & Mean & Std. deviation \\
\hline Age & 30 & 32 & 90 & 57.37 & 12.489 \\
\hline
\end{tabular}

arrangement at a different time interval (before, during, post-treatment) was done by repeated measures ANOVA. There is evidence of statistical significance among all the groups.

Table 7 reveals intergroup comparison of leukocytic infiltration scores among lesional mucosa and adjacent mucosa by Independent t-test. Statistical significance is evident in during treatment and posts radiotherapy group. No evidence of statistical significance in adjacent mucosa and lesional mucosa in pre-treatment smear.

\section{DISCUSSION}

India has one of the highest incidence of oral cancer making it the most common cancer among men (men: women ratio $2: 1$ ) and accounts for about $30 \%$ of all new cases annually. ${ }^{23}$ A recent survey of cancer mortality in India shows cancer of the oral cavity as the leading cause of mortality in men and responsible for $22.9 \%$ of cancerrelated deaths. ${ }^{24}$

The overall 5-year survival rate for all stages of oral cancer is $60 \%$. These rates are better for localized tumors as compared to tumors with regional or distant metastasis. ${ }^{25}$

Table 2: Intergroup comparison of multinucleation score among lesional mucosa and adjacent mucosa by Independent t-test

\begin{tabular}{llll}
\hline & \multicolumn{2}{c}{ Period of study } \\
\cline { 2 - 3 } & & & \multicolumn{2}{c}{$p$-value repeated } \\
Pretreatment & During treatment & Post radiotherapy \\
\hline Adjacent & $0.07 \pm .254$ & $1.1667 \pm .83391$ & $3.5333 \pm 1.25212$ \\
Lesional & $4.33 \pm 1.918$ & $14.2000 \pm 3.89872$ & $39.4000 \pm 9.69749$ \\
Mean difference & -4.267 & -13.03333 & -35.8667 \\
t-value & -12.080 & -17.905 & -20.091 \\
p-value & $<0.001$ & $<0.001$ & $<0.001$ \\
\hline
\end{tabular}

Intra group comparison for multinucleation at different time intervals (pre vs during vs post) were done by repeated measures ANOVA

Table 3: Intergroup comparison of nuclear enlargement score among lesional mucosa and adjacent mucosa by Independent t-test

\begin{tabular}{lllll}
\hline & \multicolumn{4}{c}{ Period of study } \\
\cline { 2 - 5 } & Pretreatment & During treatment & Post radiotherapy & $\begin{array}{c}\text { p-value repeated } \\
\text { measures ANOVA }\end{array}$ \\
\hline Adjacent & $1.83 \pm 1.510$ & $11.8000 \pm 4.74451$ & $14.3667 \pm 6.89569$ & $<0.001$ \\
Lesional & $26.93 \pm 4.884$ & $66.6667 \pm 9.78505$ & $97.5000 \pm 16.89675$ & $<0.001$ \\
Mean difference & -25.100 & -54.86667 & -83.13333 & \\
t-value & -26.890 & -27.635 & -24.951 & $<0.001$ \\
p-value & $<0.001$ & $<0.001$ & $<-01$
\end{tabular}

Intra group comparison for nuclear enlargement at different time intervals (pre vs during vs post) were done by repeated measures ANOVA

Table 4: Intergroup comparison of cellular enlargement score among lesional mucosa and adjacent mucosa by Independent t-test

\begin{tabular}{lllll}
\hline & \multicolumn{4}{c}{ Period of study } \\
\cline { 2 - 5 } & Pre-treatment & During treatment & Post radiotherapy & $\begin{array}{l}\text { p-value repeated } \\
\text { measures ANOVA }\end{array}$ \\
\hline Adjacent & $2.13 \pm 1.432$ & $10.8667 \pm 4.28899$ & $14.4667 \pm 6.17968$ & $<0.001$ \\
Lesional & $23.43 \pm 4.224$ & $59.7000 \pm 7.20704$ & $83.2333 \pm 7.62339$ & $<0.001$ \\
Mean difference & -21.300 & -48.83333 & -68.76667 & \\
t-value & -26.159 & -31.892 & -38.381 & $<0.001$ \\
p-value & $<0.001$ & $<0.001$ & $<$ & \\
\hline
\end{tabular}

Intra group comparison for cellular enlargement at different time intervals (pre vs during vs post) were done by repeated measures ANOVA 
Table 5: Intergroup comparison of cytoplasmic vacuolation score among lesional mucosa and adjacent mucosa by Independent t-test

\begin{tabular}{lllll}
\hline & \multicolumn{4}{c}{ Period of study } \\
\cline { 2 - 5 } & Pretreatment & During treatment & Post radiotherapy & $\begin{array}{l}\text { p-value repeated } \\
\text { measures ANOVA }\end{array}$ \\
\hline Adjacent & $0.13 \pm 0.507$ & $1.5000 \pm 2.93316$ & $2.2000 \pm 3.69902$ & $<0.001$ \\
Lesional & $2.40 \pm 0.894$ & $11.6667 \pm 1.60459$ & $39.8667 \pm 2.56949$ & $<0.001$ \\
Mean difference & -2.267 & -10.15667 & -37.6667 & \\
t-value & -2.073 & -16.655 & -45.807 & $<0.001$ \\
p-value & $<0.001$ & $<0.001$ & $<$ & \\
\hline
\end{tabular}

Intra group comparison for cytoplasmic vacuolation at different time intervals (pre vs during vs post)were done by repeated measures ANOVA

Table 6: Intergroup comparison of cytoplasmic granulation among lesional mucosa and adjacent mucosa by Independent t-test

\begin{tabular}{llll}
\hline & \multicolumn{3}{c}{ Period of study } \\
\cline { 2 - 3 } & & \multicolumn{2}{c}{$\begin{array}{c}\text { P-value repeated } \\
\text { measures ANOVA }\end{array}$} \\
\hline Adjacent & $2.20 \pm 1.095$ & During treatment & Post radiotherapy \\
Lesional & $7.47 \pm 1.852$ & $10.1000 \pm 1.47040$ & $2.2000 \pm 3.69902$ \\
Mean difference & -5.267 & $24.0000 \pm 2.85271$ & $39.8667 \pm 2.56949$ \\
t-value & -13.406 & -13.9000 & -37.66667 \\
p-value & $<0.001$ & -23.722 & -45.807 \\
\hline Intra group comparison for cytoplasmic granulation at different time intervals (pre vs during vs post) were done by repeated measures ANOVA
\end{tabular}

Intra group comparison for cytoplasmic granulation at different time intervals (pre vs during vs post) were done by repeated measures ANOVA

Table 7: Intergroup comparison of leukocytic infiltration score among lesional mucosa and adjacent mucosa by Independent t-test

\begin{tabular}{lllll}
\hline & \multicolumn{4}{c}{ Period of study } \\
\cline { 2 - 5 } & Pretreatment & During treatment & Post radiotherapy & $\begin{array}{l}\text { p-value repeated } \\
\text { measures ANOVA }\end{array}$ \\
\hline Adjacent & $30.47 \pm 2.569$ & $70.8333 \pm 5.29856$ & $100.4333 \pm 10.81724$ & $<0.001$ \\
Lesional & $32.70 \pm 8.133$ & $78.4667 \pm 17.90537$ & $109.0333 \pm 13.17648$ & $<0.001$ \\
Mean difference & -2.233 & -7.6333 & -8.6000 & \\
t-value & -1.434 & -2.239 & -2.763 & \\
p-value & 0.157 & 0.032 & 0.008 & \\
\hline
\end{tabular}

Intra group comparison for leukocytic infiltration at different time intervals (pre vs during vs post) were done by repeated measures ANOVA.

All three main treatment modalities-surgery, radiation (RT), and chemotherapy are used to treat oral cancer, either alone or in combination. ${ }^{26}$

Cellular alterations caused by radiation exposure can be linked to the damage that leads to mitotic cell death. Cell division is initiated and controlled by the centrioles and the pericentriolar matrix (PCM). Radiation-induced peroxidation of lipids in the cell membrane can cause structural and functional alteration to it. ${ }^{27}$

For oral cancer, cytology has been an option and proved to be a consistent primary diagnostic test. It can also be of value where a surgical biopsy is not indicated or in post-radiotherapy follow-up cases. The combined histological and cytological assessment of a lesion has been found to give the highest percentage of early diagnosis of oral cancers. ${ }^{28}$

Multinucleation: In the research conducted by Silverman et al., multinucleation was the most common radiation-induced change in oral cancers. These findings were later confirmed by many researchers. ${ }^{17}$

Mehrotra and his colleagues observed that in normal mucosa and malignant cells, the frequency of multinucleation was increased with increased radiotherapy dosage in serial scrape smears from both sides. They also reported a significant association between multinucleation on normal mucosa and malignant cells and radiation dose $(p<0.001){ }^{29}$

Before treatment, Bhattathiri et al. observed that the mean multinucleated count was 3.7/1000 mononucleated cells. With radiation, the frequency of cells with multinucleation increased significantly $(p<0.0001)$ to a maximum of $16.8 / 1000$ mononucleated cells. ${ }^{30}$ Raj et al. in their study found out that multinucleated cells were significantly increased from 0.1 per thousand cells to 0.9 at 24 Gy $(p=0.05)$ on the normal side and from 0.1 per thousand to 1.2 at $24 \mathrm{~Gy}(\mathrm{p}=0.01)$ on the lesional side. ${ }^{14}$

Bhattathiri et al. in their study stated as the fact that multinucleation showed the greatest relationship with radiosensitivity, suggesting that injury to the cytokinetic apparatus is important in determining tumor radiosensitivity. ${ }^{31}$ The findings of our study depict a dose-related change in the number of multinucleated cells which has a highly significant increase in both normal as well as malignant cells. These findings were in accordance with above-mentioned studies. 


\section{Nuclear Enlargement}

Bindu et al. stated that there was no significant difference in the nuclear shape in relation to radiation dose. ${ }^{19}$ Ogden et al. found significant rise in nuclear area resulting from exposure to irradiation (before treatment compared with halfway through treatment). ${ }^{32}$ Mehrotra et al. in their study observed that as the fractions of the radiotherapy increased, the relative size of the nucleus and nuclear area also increased. ${ }^{33}$

An increase in nuclear size after undergoing irradiation was also reported in the present study, a finding which was in accordance with Ogden et al. ${ }^{32}$ and Mehrotra et al. ${ }^{33}$

\section{Cellular Enlargement}

Hannah Peters noted an increase in cell size in normal squamous epithelium which received radiation along with cancerous epithelium. ${ }^{34}$ Ogden et al. found significantly rise in the cellular area resulted from exposure to irradiation (before treatment compared with halfway through treatment). ${ }^{32}$

In the present study, the mean value of the lesional and adjacent mucosa showed a significant rise from the pretreatment value to the post-radiotherapy. The results of our study are in accordance with the results of studies conducted by Peters ${ }^{34}$ and Ogden et al. ${ }^{32}$

The results of our study are not in accordance with the study conducted by a study conducted by Silverman et al. ${ }^{17} \mathrm{He}$ found that in regard to each specific cellular morphologic change, the frequency of enlarged cells increased slightly after the beginning of irradiation. The frequency of cell enlargement showed no consistent relationship to clinical response to neoplasms.

\section{Cytoplasmic Vacuolation}

The percentage of the cells showing cytoplasmic vacuolation increased from the pretreatment value of 4.7 to $8.7 \%$ in a study conducted by Bindu et al. However, this increase in value was not significant. ${ }^{19}$ According to Silverman et al. no change in the degree of vacuolization was observed in patients post radiotherapy. ${ }^{17}$ The results of our study were contradictory with the findings of studies done by Bindu et al. ${ }^{19}$ and Silverman et al. ${ }^{17}$

Agarwal et al. stated that exfoliative cytology of oral squamous cell carcinoma patients undergoing fractionated radiotherapy showed that with increasing dose of radiation, the number of cells exhibiting cytoplasmic vacuolation increased. ${ }^{35}$ The results of our study are in accordance with this study.

\section{Cytoplasmic Granulation}

Hannah Peters stated that the appearance of the cytoplasmic granules in the superficial squamous cells is the most constant microscopic finding after radiation of the squamous epithelium of the mouth. ${ }^{34}$ According to Mehrotra et al. cytoplasmic granulation has not been reported in previous studies conducted in patients undergoing fractionated radiotherapy. They found that there was a non-specific change in the cytoplasm comprising of cytoplasmic granulation, which was found in both normal and malignant cells. The number was higher in malignant cells and increased with duration of radiation. $^{33}$

Mehrotra and his colleagues observed a significant dose-related increase in cytoplasmic granulation count in both normal and malignant cells. The appearance of cytoplasmic granules in the squamous cells is the most constant microscopic finding after irradiation of oral squamous epithelium. ${ }^{29}$ In the current study, there was a significant dose-related increase in the cytoplasmic granulation in both lesional and adjacent mucosa. The results of our findings were in accordance with abovementioned studies.

\section{Leukocytic Infiltration}

In the present study, the p-value of repeated measure analysis of variance (ANOVA) and post hoc test was statistically significant in both lesional and adjacent mucosa at different time intervals. But on a comparison of the values of adjacent mucosa and lesional mucosa by Independent $t$-test, the $p$-value in the pre-treatment smears was not found to be statistically significant.

No significant increase in the extent of neutrophils was observed following radiation exposure in a study conducted by Bindu et al. ${ }^{19}$ Agarwal et al. observed that with the increase in dose of radiation there was an increase in the mean percentage of leukocytic infiltration from pre-treatment smear to four weeks post-radiotherapy. ${ }^{35}$ Ahmed and Elemirri found increased inflammatory infiltrates in posttherapeutic smears, as compared to non-treated patients. This suggests the role of radiotherapy in inducing inflammatory changes. ${ }^{36}$

Though, among all the parameters considered in the study, leukocytic infiltration was the only finding found, which was not statistically significant in terms of pretreatment smears on a comparison of the values of adjacent mucosa and lesional mucosa by independent $t$-test.

The major limitation of this study was sample collection from the unapproachable lesional areas. In addition number of cases is low due to a significant dropout rate of many patients. 


\section{CONCLUSION}

The findings of the present study emphasize that various nuclear and cellular abnormalities reveal a statistically significant increase with increasing radiation doses and time interval in adjacent normal mucosa as well as lesional mucosa. Knowledge about the sensitivity of the different treatment modalities for oral cancer treatment will greatly benefit judicious treatment planning for these patients. A possible role of the predictive value of these changes deserves further evaluation in a large multi-institutional study.

\section{REFERENCES}

1. Sugerman PB, Savage NW. Exfoliative cytology in clinical oral pathology. Australian dental journal. 1996 Apr 1; 41(2):71-74.

2. Beale LS. Examination of sputum from a case of cancer of the pharynx and the adjacent parts. Arch Med. 1860;2(44):1860-1861.

3. Lebert H. Traite pratique des maladies cancereuses. JB Baillière; 1851.

4. Papanicolau GN. Atlas of exfoliative cytology. Cambridge, Mass. Harvard University Press. 1954.

5. Papanicolaou GN, Traut HF. Diagnosis of uterine cancer by the vaginal smear. New York. 1943;46.

6. Ayre JE. Cancer cytology of the uterus: introducing a concept of cervical cell pathology. Churchill; 1951.

7. Montgomery PW. A study of exfoliative cytology of normal human oral mucosa. Journal of dental research. 1951 Feb 1; 30(1):12-18.

8. Koss LG, Melamed MR, editors. Koss' diagnostic cytology and its histopathologic bases. Lippincott Williams \& Wilkins; 2006.

9. Warnakulasuriya S. Global epidemiology of oral and oropharyngeal cancer. Oral oncology. 2009 May 31;45(4):309-316.

10. Zheng JW, Qiu WL, Zhang ZY. Combined and sequential treatment of oral and maxillofacial malignancies: an evolving concept and clinical protocol. Chinese medical journal. 2008 Oct;121(19):1945-1952.

11. Arneson AN, Stewart FW. Clinical and histologic changes produced in carcinoma of the cervix by different amounts of roentgen radiation: a comparison. Archives of Surgery. 1935 Oct 1;31(4):542-567.

12. Graham RM. The effect of radiation on vaginal cells in cervical carcinoma; the prognostic significance. Surgery, gynecology \& obstetrics. 1947 Feb;84(2):166-173.

13. Shier CB. The prognosis of carcinoma of the cervix uteri by vaginal smear: A preliminary report. American journal of obstetrics and gynecology. 1954 Feb 28;67(2):286-292.

14. Grossman MH, Lochte WP, Coulter Sr WW. Prognostic significance of the vaginal smear after irradiation of uterine carcinoma. Texas state journal of medicine. 1948 Dec;44(8):594-596.

15. Umiker W, Lampe I, Rapp R, Latourette H, Boblitt D. Irradiation effects on malignant cells in smears from oral cancers. A preliminary report. Cancer. 1959 May 1;12(3):614-619.

16. Silverman S, Sheline GE. Effects of radiation on exfoliated normal and malignant oral cells. A preliminary study. Cancer. 1961 May 1;(3):587-96.

17. Silverman S, Sheline GE, Gillooly CJ. Radiation therapy and oral carcinoma. Radiation response and exfoliative cytology. Cancer. 1967 Aug 1;20(8):1297-1300.

18. Wachtel EG. Radiation changes in vaginal smears. Exfoliative Cytology in Gynecological Practice. 1964:175-193.
19. Bindu L, Balaram P, Mathew A, Remani P, Bhattathiri VN, Nair MK. Radiation-induced changes in oral carcinoma cells-a multiparametric evaluation. Cytopathology. 2003 Oct 1;14(5):287-293.

20. Shield PW, Wright RG, Free K, Daunter B. The accuracy of cervicovaginal cytology in the detection of recurrent cervical carcinoma following radiotherapy. Gynecologic oncology. 1991 Jun 30;41(3):223-229.

21. Cowpe JG, Longmore RB, Green MW. Quantitative exfoliative cytology of abnormal oral mucosal smears. Journal of the Royal Society of Medicine. 1988 Sep 1;81(9):509-513.

22. Raj V, Mahajan S. Dose response relationship of nuclear changes with fractionated radiotherapy in assessing radiosensitivity of oral squamous cell carcinoma. Journal of Clinical and Experimental Dentistry. 2011;3(3):e193-200.

23. Sankaranarayanan R, Ramadas K, Thomas G, Muwonge R, Thara S, Mathew B, et al. Effect of screening on oral cancer mortality in Kerala, India: A cluster-randomised controlled trial. Lancet 2005;365:1927-1933.

24. Dikshit R, Gupta PC, Ramasundarahettige C, Gajalakshmi V, Aleksandrowicz L, Badwe R, et al. Cancer mortality in India: A nationally representative survey. Lancet 2012;379:1807-1816.

25. Ferlay J, Shin HR, Bray F, Forman D, Mathers C, Parkin DM. GLOBOCAN 2008 v2.0, Cancer incidence and mortality worldwide: IARC CancerBase No. 10. Lyon, France: International Agency for Research on Cancer; 2010.

26. Prelec J, Laronde DM. Treatment modalities of oral cancer. Canadian Journal of Dental Hygiene. 2014 Feb;48(1):13-19.

27. Mehrotra R, Singh M. Serial scrape smear cytology of radiation response in normal and malignant cells of oral cavity. Indian journal of pathology \& microbiology. 2004 Oct;47(4): 497-502.

28. Minhas S, Kashif M, Nagi AH. Evaluation of Various Nuclear Cytological Changes in Normal Buccal Mucosa and Peritumoural Area in Patients with Oral Squamous Cell Carcinoma Receiving Concomitant Chemoradiotherapy. Pathology research international. 2016 Apr;11;2016.

29. Mehrotra R, Singh M. Serial scrape smear cytology of radiation response in normal and malignant cells of oral cavity. Indian journal of pathology \& microbiology. 2004 Oct;47(4):497-502.

30. Bhattathiri NV, Bindu L, Remani P, Chandralekha B, Nair KM. Radiation-induced acute immediate nuclear abnormalities in oral cancer cells. Acta cytologica. 1998 Jul 1;42(5):1084-1090.

31. Bhattathiri NV, Bharathykkutty C, Prathapan R, Chirayathmanjiyil DA, Nair KM. Prediction of radiosensitivity of oral cancers by serial cytological assay of nuclear changes. Radiotherapy and oncology. 1998 Oct 31;49(1):61-65.

32. Ogden GR, Cowpe JG, Green MW. Effect of radiotherapy on oral mucosa assessed by quantitative exfoliative cytology. Journal of clinical pathology. 1989 Sep 1;42(9):940-943.

33. Mehrotra R, Goel N, Singh M, Kumar D. Radiation-related cytological changes in oral malignant cells. Indian journal of pathology \& microbiology. 2004 Jul;47(3):343-347.

34. Peters H. Cytologic smears from the mouth: Cellular changes in disease and after radiation. American journal of clinical pathology. 1958 Mar 1;29(3):219-225.

35. Agarwal D, Khan N, Siddhiqu SA, Afroz N. Assessment of Various Cytological Changes For Predicting Radiosensitivity of Oral Cavity Cancer by Serial Cytology. JK Science 2011;13(4):171.

36. Ahmed HG, Elemirri DA. Assessment of oral cytological changes associated with exposure to chemotherapy and/or radiotherapy. Cy to Journal. 2009 Jan 1;6(1):8. 